\title{
Combination Antifungal Therapy: When, Where, and Why
}

\author{
Elizabeth S. Belanger ${ }^{1} \cdot$ Eric Yang ${ }^{1} \cdot$ Graeme N. Forrest $^{2}$
}

Published online: 31 March 2015

(C) Springer International Publishing AG 2015

\begin{abstract}
Combination antifungal therapy in the treatment of invasive fungal infections remains a controversial topic despite its increasing use based off case reports and series. There remains a paucity of well-controlled clinical studies to address which combination, the timing, and the benefits to the underlying host without causing toxicity. The evidence for using amphotericin B with 5 flucytosine for cryptococcal infections to reduce mortality has been effectively proven but the inaccessibility to 5 flucytosine requires the utilization of less effective combinations in many countries. For invasive aspergillosis, a large controlled clinical study in hematologic malignancy patients showed a trend for combination of voriconazole and anidulafungin to reduce mortality, especially in a subgroup of patients with positive galactomannan results. Outside of these two indications, the use of combination antifungal therapy in other patients and those with rare mold
\end{abstract}

This article is part of the Topical Collection on Mycology

Graeme N. Forrest

forrestg@ohsu.edu

Elizabeth S. Belanger

belanger@ohsu.edu

Eric Yang

yaner@ohsu.edu

1 Division of Infectious Diseases, Oregon Health and Science University, 3181 SW Sam Jackson Park Rd, Portland, OR 97239, USA

2 Division of Infectious Diseases, Portland Veteran Affairs Medical Center, 3710 SW US Veterans Hospital Rd, P3-ID, Portland, OR 97239, USA infections has not been proven and outcomes are heavily influenced by host factors rather than the combinations of antifungal treatment selected.

Keywords Combination antifungal therapy $\cdot$ Aspergillus . Voriconazole $\cdot$ Echinocandin $\cdot$ Cryptococcus

\section{Introduction}

The role of when, where, and why of combination antifungal (CAF) therapy has been debated for almost two decades and outside of cryptococcal meningitis remains controversial. The rationale for combination antifungal therapy has mostly been targeted at the invasive mold infections such as aspergillosis and mucormycosis where morbidity and mortality have traditionally been high. The population who develops these invasive fungal infections (IFI) usually have significant host factors that will affect clinical responses to treatment of their IFI. They are usually immunocompromised with processes such as prolonged neutropenia, solid organ transplantation (SOT), or graft versus host disease (GvHD) from stem cell transplantation (SCT), and outcomes of their IFI treatment may be unrelated to the antifungal therapy used. Given these host issues, CAF therapy remains a potential potent option for treatment of severe IFI.

CAF therapy approaches are utilized on the presumption that they will broaden antifungal coverage, be synergistic in antifungal activity, become more fungicidal, minimize the risk of development of resistance, and lastly decrease toxicity [1, 2]. These reasons are very appealing given the complicated host factors and often the difficulty in obtaining fungal cultures and species identification in a rapid timeframe in which a delay in effective therapy may result in progression of the 
fungal disease. As a result, the use of CAF therapy using agents with different targets and spectrum of activities has become an increasing practice, especially for the empiric treatment of suspected IFI [1]. Lastly, the benefit of CAF therapy becomes an option of last resort in fungal infections where there is high intrinsic resistance to current antifungal agents. However, there may be unintended consequences for using CAF therapy. These include the possibility that the combination may attenuate each other's activity, increase resistance, increase toxicity and drug interactions, and lastly increase costs without any known benefit to the patient [1].

This review will evaluate recent clinical and in vitro/vivo data on combination antifungal therapy to see if we can answer the when, where, and why to use this treatment strategy $[1,3]$. The areas to review will be based on the fungal infections most commonly encountered in clinical practice.

\section{Aspergillosis}

Serious invasive infections from Aspergillus species are usually in patients with significant host defense weaknesses, in particular patients with hematologic malignancies, significant neutropenia, GvHD, and organ and stem cell transplantation recipients [4]. There is little evidence to discuss CAF therapy for invasive Aspergillus (IA) outside of these host factors, and the focus will be on the high-risk hosts for invasive disease and not for aspergillomas. IA is a common complication in patients with hematologic malignancies and SCT and is associated with a mortality rate over $20 \%[5 \bullet \cdot$. Similarly in SOT, IA is associated with high mortality (9-20\%) and morbidity, primarily complicated by drug interactions and allograft dysfunction [6]. The introduction of voriconazole has reduced the rate of mortality and improved outcomes for the treatment of IA, but mortality remains high. Subsequently, combining this antifungal agent with others has become an increasingly common practice, especially in salvage settings where the patients have clinically failed antifungal monotherapy and CAF therapy was started $[7,8 \bullet]$. Unfortunately, for many years, data on the optimal CAF therapy for the treatment of IA has been limited to neutropenic animal models, case reports, and single-center case series [8•]. As a result, these data are of limited use clinically with such a large reporting bias of positive reports and the true efficacy of outcomes largely unknown. The greatest limitation of many of the prior CAF studies for IA treatment have been that they are salvage studies where other factors may have been involved in clinical responses (neutropenia resolution, reduction in immunosuppression, resolution of drug toxicity) and, in fact, patients may have eventually succeeded with monotherapy. Also, many of the reports on CAF therapy for IA have actually been sequential CAF therapy (initially started as monotherapy and then second agent added later) rather than primary or initial CAF therapy $[8 \bullet, 9 \bullet, 10 \bullet]$. As such a review of recent preclinical and clinical IA CAF studies with use of amphotericin B products, echinocandins or triazoles will follow.

\section{Preclinical Studies}

There are numerous in vitro and in vivo studies combining two antifungal agents over the last 15 years [9॰]. The combinations of triazole with echinocandin or triazole with amphotericin B appear to be the most common combinations tested [11-17]. The problem with in vitro studies is determining the likely clinical efficacy as there are currently no current standards for testing combined antifungal agents [18]. Therefore, there are a mixture of synergy and antagonism reported and clinical outcomes based on susceptibility testing in molds has not shown the same success as with yeasts [19]. There appears to be more reliable information from in vivo studies for IA with CAF therapies [12, 20-22]. Two recent in vitro studies again suggest in experimental pulmonary IA that an echinocandin with triazole appears to be highly synergistic $[14,22]$. Zhang et al. demonstrated that the combination of caspofungin with voriconazole in an experimental pulmonary IA study using neutropenic rats resulted in significantly prolonged survival and reduction in fungal burden compared to caspofungin monotherapy [22]. This result is not surprising given that caspofungin monotherapy has shown clinical breakthroughs in the neutropenic host, so this study adds very little benefit to the use of combination therapy argument and reinforces the role of voriconazole [23]. A new triazole, isavuconazole, was tested with micafungin against most medically important molds, including Aspergillus species, and in vitro synergy was obtained; however, again this does not support their combined use.

\section{Clinical}

The lack of high quality clinical trials to determine the benefits of CAF therapy for IA has been a vexing issue. There have been 10 registered clinical studies since 2001 with until recently only two publishing results. The rest either closed prematurely due to lack of enrollment or were not published. The two studies that did complete and publish were open-labeled non-randomized studies with selection bias and all these studies are summarized by Martin-Pena [9•]. Finally, this lack of any clinical trial data has been corrected with the recent study evaluating voriconazole versus voriconazole with anidulafungin $[5 \cdot \bullet]$. This multicenter, international study is the first randomized, double-blinded trial comparing monotherapy to combination therapy. The study addressed many of the limitations of previous open-labeled studies by only enrolling a high-risk population with hematologic malignancies and stratifying the randomization based on presence of an allogeneic SCT. Patients were enrolled based on possible, 
probable, or proven IA based on consensus guidelines, but possible IA had to be upgraded based on microbiologic or use of either serum or bronchoalveolar (BAL) galactomannan (GM) results within a week to be counted [5*0]. Patients had to receive at least 2 weeks of anidulafungin or placebo and 6 weeks of high dose voriconazole ( $300 \mathrm{mg}$ bid orally) after intravenous loading for analysis. Their primary endpoints were all cause mortality at 6 weeks and secondary endpoints were all cause mortality at 12 weeks and within subgroups (i.e., SCT, GvHD, neutropenia). Other endpoints included radiologic response at 6 weeks as adjudicated by independent committee. The study was powered to show superiority of CAF therapy over monotherapy with a presumed reduction in mortality from 19.6 to $7 \%$ [5*0]. They were able to analyze 277 out of 454 eligible patients with proven or probable IA. The groups were well balanced with about $30 \%$ in each group with allogeneic SCT and $60 \%$ neutropenic at study entry. Overall, $80 \%$ had a proven diagnosis of IA at baseline with either proven culture or GM result. The study outcomes are very compelling for CAF therapy in the hematologic patient population. Comparing monotherapy to CAF therapy, overall mortality was 27.8 vs $19.3 \%$ (treatment difference $(\mathrm{TD})=-8.5 \%$, confidence intervals $(\mathrm{CI})-19$ to $1 \%$ ) and at 12 weeks 39.4 vs $29.3 \%$ (TD $=-10.1 \%$, CI -21.4 to $1.1 \%$, $p=0.077$ ). No significant differences in overall mortality were seen when stratified for allogeneic SCT or neutropenia duration between monotherapy and CAF therapy groups. When specifically looking at IA attributable mortality, there also was no significant between the two groups ( 88.5 vs $84.6 \%)$. This study performed a subanalysis and post-hoc analysis, and identified a beneficial observation for CAF therapy for both global outcomes and mortality after 6 weeks. This was an unusual finding as it occurred after completion of all the antifungal therapy. However, on subanalysis patients with an elevated GM, result at baseline appeared to have reduced overall mortality in CAF therapy (15.7 \%) vs monotherapy (29.3\%) $(p=0.037)[5 \bullet \cdot$. This study was well constructed and answered many difficult questions about study design and clinical outcomes measured in fungal studies. The positives included the use of a homogenous population, powering the study for a meaningful endpoint of clinical superiority and demonstrating that the traditional global response composite endpoints so often used in antifungal studies may result in incorrect interpretation of results due to bias and subjectivity. Also, they showed that the combination of high dose voriconazole and anidulafungin was a very safe combination therapeutically in this population. Unfortunately, the study did not show a significant benefit for CAF therapy for the treatment of IA in hematologic malignancy patients; however, there appears to be a subset of patients where clinicians could consider CAF therapy - patients with elevated baseline GM levels (either serum or BAL). Given the strength of the data, this study gives compelling reason for hematologic malignancy patients with baseline elevated GM results to start CAF therapy in primary rather than as salvage therapy. The benefits of this strategy are that the GM test returns earlier and is easier to obtain than cultures and thus one can initiate directed therapy earlier in a targeted population. This strategy addresses concerns of widespread use of unnecessary CAF therapy and satisfies concerns for undertreatment of a serious IA infection in a particularly high-risk patient population.

A summary of other data published over the last 4 years is presented in Table 1. For IA, many are monocentric studies without controls or meta-analyses of these poorly performed studies. Interpreting the data can be difficult due to the variability in patient populations, problems with definitions of disease, and whether they are primary treatment or salvage therapy. For instance, a meta-analysis evaluated the use of either triazoles or amphotericin B with an echinocandin for IA salvage therapy [8•]. This analysis evaluated studies with primary outcomes of mortality at 12 weeks within the literature. From over 3,000 papers, they were only able to analyze 16 studies ( 2 clinical trials and 14 observational studies) [8•]. This meta-analysis could not find any support for CAF as primary therapy, but many of these observational, salvage studies showed CAF having a benefit over monotherapy (odds ratio $=1.8($ CI 1.08-3.01) $)$. Unfortunately, this analysis is limited again by selection biases of these observational studies and lack of randomization biases toward the treatment effect. A high dose caspofungin combination therapy study looked mostly at the safety data rather than the clinical outcomes [24•]. This study used $100 \mathrm{mg}$ caspofungin daily with voriconazole in hematologic malignancy patients with proven or probable IA of which $50 \%$ had undergone SCT. Despite the use of CAF therapy, 40 of 91 patients died of IA, with risk factors identified for poor outcomes including high dose corticosteroids and intensive care unit (ICU) stay [24•]. The investigator also noted that CAF therapy was also limited by drug-induced hyperbilirubinemia in their review. Thus, given these outcomes, this CAF therapy does not present an appealing treatment option in this population.

The data on the use of CAF therapy in SOT is even more limited. An early report was somewhat favorable to the combination of voriconazole and caspofungin. In a prospective multicenter observational study in IA with SOT, it was found that the CAF treatment group was not different in survival compared to a control group of lipid formulations of amphotericin B (AmB) treatment. However, CAF therapy was superior in those with renal failure and those with Aspergillus fumigatus infection [25]. Furthermore, the only recent CAF data are for the treatment of IA in liver transplantation recipients. Barschiesi et al. reviewed treatment in 116 liver transplant recipients over a 28 -year span with proven IA [26•]. CAF therapy with either amphotericin B or voriconazole with an echinocandin was used in $51 \%$ of their cases. These investigators showed that both voriconazole 


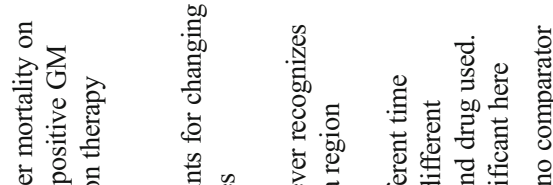

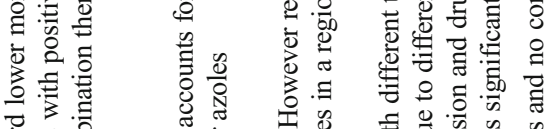

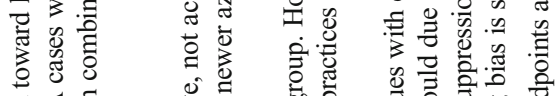

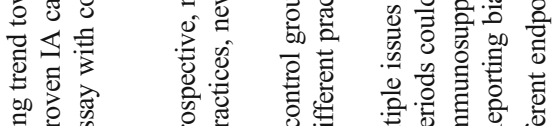

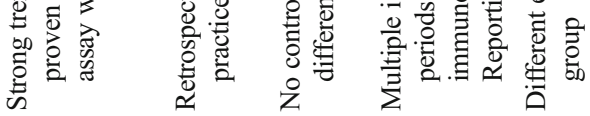
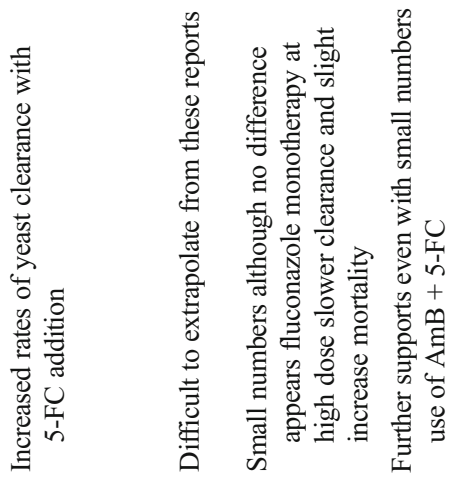

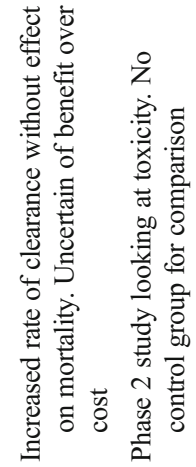

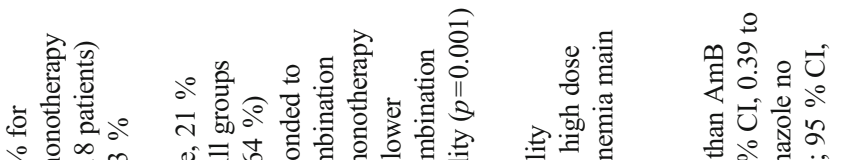

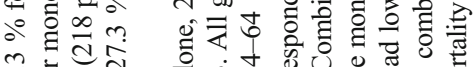

व.

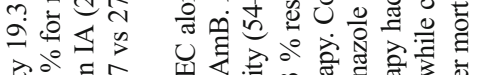

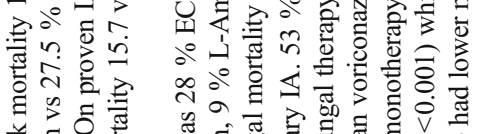

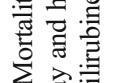

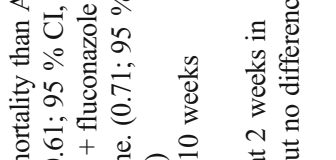

$\mathrm{O}=0$

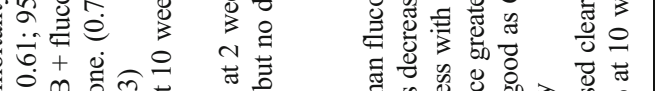

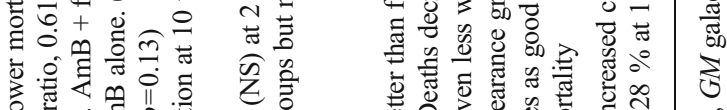

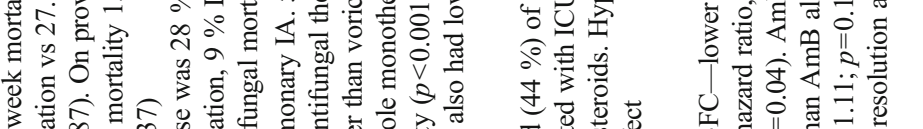

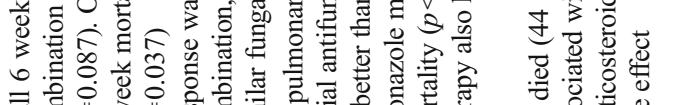

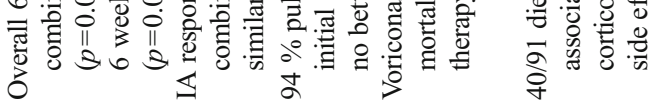

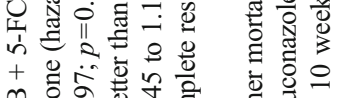

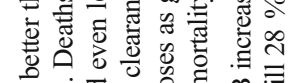

帘品

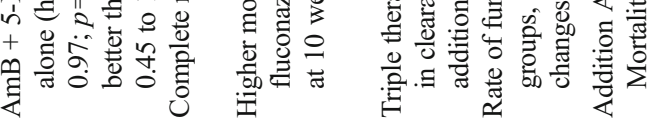

:

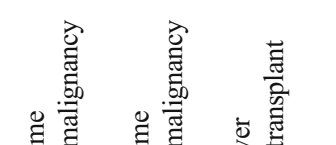

䓫

茜 递

穿

过 :

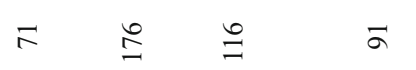

范

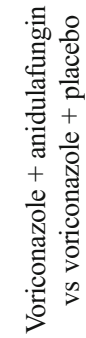

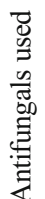

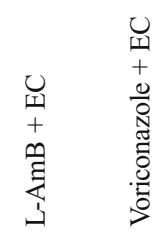

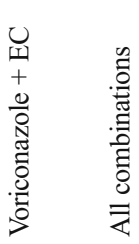

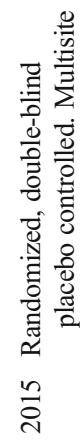

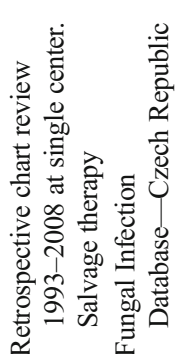

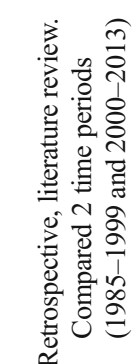

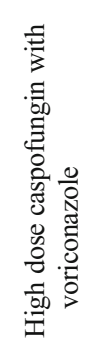

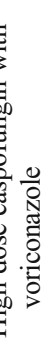

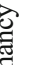

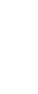

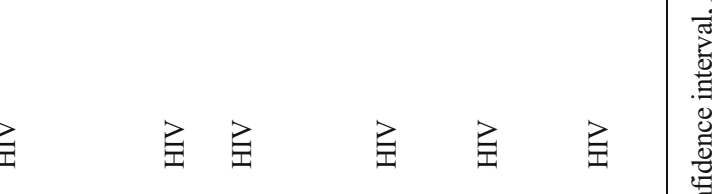

ते $\quad$ क \& क

U

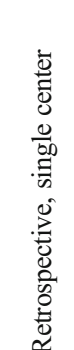

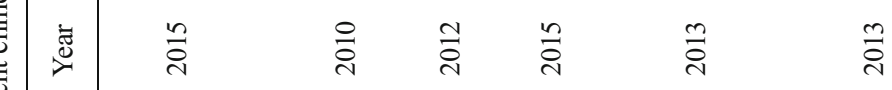

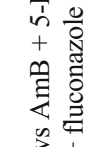

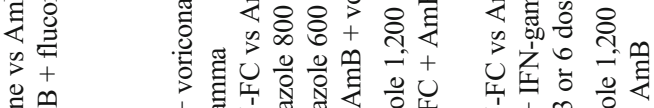

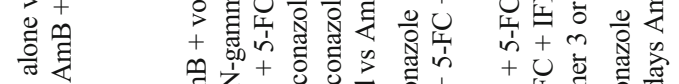

安

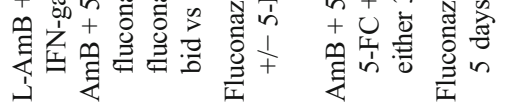

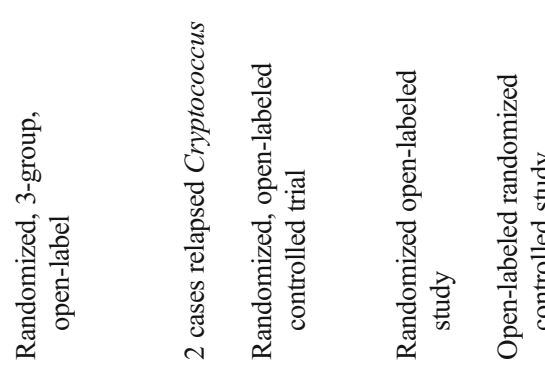

$\approx$

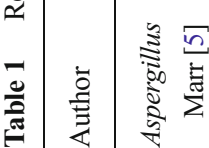

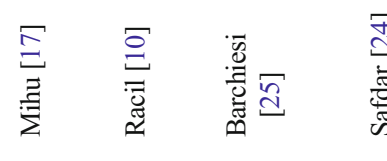

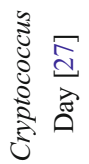

วั

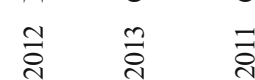

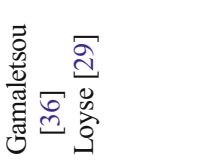

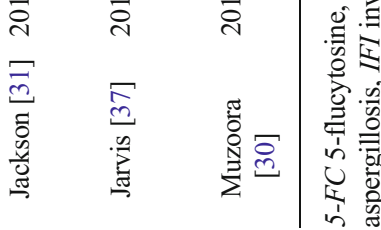


monotherapy $(p<0.001)$ and CAF therapy $(p<0.001)$ were associated with improved patient survival [26•]. There are many limitations of this study, including the analysis in not accounting for era differences in management of immunosuppression, rejection, and newer antifungal agents. Also, there is no analysis on disease location (pulmonary or central nervous system) and the specific outcomes with each treatment. Although a well-intentioned study, not accounting for changes in practice and management techniques, can lead to faulty interpretations, and as such given, the success of voriconazole monotherapy in their data does not encourage the use of CAF therapy with its potential drug-drug interactions and costs in this population.

In summary, after many years, there slowly appears to some clarity in the role of CAF therapy for IA most explicitly for patients with hematologic malignancies and an elevated GM result. It appears that CAF with voriconazole with an echinocandin are the preferred regimens and there are no recent data on amphotericin B products with other antifungals. Outside of this indication for IA, there is a lack of strong clear data and most likely CAF therapy will be dictated by individual prescriber preferences and mostly in salvage and/or severe situations.

\section{Cryptococcus}

The Infectious Diseases Society of America (IDSA) guidelines for the management of cryptococcal infections recommend that $\mathrm{AmB}$ deoxycholate, or a lipid formulations (L$\mathrm{AmB})$, combined with 5-flucytosine (5-FC) is the first line induction therapy for cryptococcal meningoencephalitis in both immunocompetent and immunocompromised patients [27]. The strength of evidence for the combination of $\mathrm{AmB}$ product with 5-FC for the treatment of cryptococcal infections has recently been reinforced by a large clinical study in VietNam in human immunodeficiency virus (HIV)-infected patients. Day et al. performed an open-labeled randomized clinical study comparing $\mathrm{AmB}(1 \mathrm{mg} / \mathrm{kg} /$ day $)$ alone to $\mathrm{AmB}+5-$ $\mathrm{FC}(100 \mathrm{mg} / \mathrm{kg} /$ day $)$ and to AmB + fluconazole (400 mg bid) for 2 weeks and then fluconazole consolidation/maintenance therapy $[28 \cdot \bullet]$. Outcomes were all cause mortality at 14 and 70 days after starting therapy. The results were rather drastic with $\mathrm{AmB}+5-\mathrm{FC}$ showing significantly reduced risk of death at 70 days (hazard ratio 0.61, CI $0.39-0.97, p=0.04$ ) and combination with fluconazole also having lower mortality but not significant to $\mathrm{AmB}$ alone and this survival benefit was supported by an improved early fungicidal activity (EFA) [28••]. This survival advantage of $\mathrm{AmB}+5-\mathrm{FC}$ continued and was more significant at 6 months than either of the other two groups $(p=0.01)$. However, there were more toxicities in the 5 -FC group with neutropenia and anemia [28••]. The effects of antiretroviral therapy impacting the results could be not determined as the numbers were too small in each group. With this data, it strongly supports the role of $\mathrm{AmB}+5$-FC for the treatment of central nervous system (CNS) cryptococcal infections, especially in HIV-infected patients.

A meta-analysis by Yao et al. has attempted to reinforce the use of either 5-FC or fluconazole combined with AmB [29]. Their data identified four prospective randomized studies that demonstrated HIV-infected patients who received AmB + 5FC had a $44 \%$ reduction in mortality and improved EFA of their cerebrospinal fluid (CSF) with this combination compared to AmB or fluconazole monotherapy [29]. However, the beneficial effect was transient, and at 3 months after starting induction therapy, there were no differences between using 5-FC or fluconazole when used in combination with AmB. Importantly, in the AmB plus fluconazole combinations, higher fluconazole doses of at least $800 \mathrm{mg}$ daily were associated with better outcomes compared to lower fluconazole doses [29].

Despite the strong evidence supporting the use of 5-FC in cryptococcal infections, 5-FC is currently unregistered and unavailable in most of Africa and Asia, despite the production of bio-equivalent compounds [30]. As such, combining fluconazole with $\mathrm{AmB}$ is often employed to address this lack of access to 5-FC, and although less effective immediately, CAF therapy with $\mathrm{AmB}$ and fluconazole appears at higher doses to be useful in these resource-limited settings [29-32]. With LAmB formulations rarely available outside Western countries and the high toxicity of conventional AmB especially with renal dysfunction, short courses of $\mathrm{AmB}$ with high doses of fluconazole $(1,200 \mathrm{mg} /$ day) have been employed and demonstrated rapid EFA in the CSF, and little toxicity but no clinical outcomes data has been included [32]. The CAF therapy of fluconazole +5 -FC as an alternative to $\mathrm{AmB}$ regimens was studied by Milefchik. They utilized a dose-ranging study in 89 patients using fluconazole doses up to $2,000 \mathrm{mg} /$ day combined with standard doses of 5-FC in HIV-associated cryptococcal meningitis [33]. In fluconazole monotherapy, higher doses of fluconazole were found to convey a survival benefit and decreased the amount of time for CSF clearance of the organism. Furthermore, the CAF addition of 5-FC to fluconazole, an all oral regimen, was found to improve response to treatment [33].

Although Cryptococcus neoformans is the most common infecting yeast, the emergence of Cryptococcus gattii, has been increasingly seen mostly in the northwestern USA and Canada, in primarily immunocompetent patients [34]. Some of these $C$. gattii infections have been described as fluconazole resistant, and use of CAF therapy with $\mathrm{AmB}+5-\mathrm{FC}$ or a triazole may be required for treatment based on susceptibility testing [35, 36, 37 •]. A small series of $C$. gattii infections in SOT recipients in Oregon showed a high attributable mortality at 120 days (45\%). Most of these patients had not received 5FC induction therapy and the VGII strain was associated with 
elevated fluconazole minimal inhibitory concentrations [37•]. Furthermore, of the new triazoles, there are only limited nonclinical data with voriconazole. The CAF of voriconazole and AmB was studied in immunodeficient mice infected with C. neoformans, and voriconazole plus $\mathrm{AmB}$ was found to prolong survival and decrease colonization of infected organs [38]. Finally, the addition of the immune modulator, interferon- $\gamma(\mathrm{IFN}-\gamma)$, to AmB and 5-FC to enhance rapid sterilization of the CSF has been proposed [39]. A phase 2 study comparing two doses of IFN- $\gamma$ to conventional therapy with $\mathrm{AmB}$ and 5-FC was studied in HIV-infected patients with cryptococcal meningitis. The addition of IFN- $\gamma$ resulted in rapid EFA in the CSF without any adverse safety effects but without any significant impact on mortality between the groups [39]. Gamaletsou et al. also described the successful salvage therapy of L-AmB, voriconazole, and IFN- $\gamma$ in treating refractory cryptococcal meningitis in two HIV patients. Both patients had not responded to initial induction therapy with prolonged $\mathrm{AmB}$ and 5-FC treatment and did well with this CAF therapy [40].

CAF therapy with AmB plus 5-FC remains the cornerstone of treatment for cryptococcal disease, especially in HIVinfected patients, and remains strongly recommended unless access to 5-FC is an issue, where fluconazole can be substituted. Outside of HIV, in transplantation and normal hosts, older data continues to support the use of CAF AmB plus 5-FC therapy [27].

\section{Other Fungal Infections}

The role of CAF therapy in other rarer infections is less known, with much of the data based on case reports or case series.

\section{Yeast}

There has been very little progress or new information since the CAF study for candidemia [41]. This study compared nonneutropenic patients receiving CAF therapy with fluconazole plus AmB compared to fluconazole plus placebo in a randomized, blinded study of 219 patients. Patients in the fluconazole plus AmB group experienced a higher rate of renal dysfunction compared to those receiving fluconazole alone (24 vs $3 \%$ ). Further analysis revealed that the largest benefit of combination therapy was seen in the midrange of APACHE II scores, suggesting that patients who were sicker did equally poorly and patients who were less sick did equally well regardless of CAF or monotherapy, and there was no mortality benefit for either treatment group [41]. Since this study, the emergence of the highly effective and safer echinocandins has rendered CAF therapy for candidemia effectively redundant.
However, with the increasing incidence of invasive resistant Candida infections, some investigators are using antibiotics or biologics with either intrinsic antifungal activity or synergistic interactions with existing antifungals [42]. Liu et al. have reviewed many studies using fluoroquinolones, tetracyclines, and calcineurin inhibitors in combination with fluconazole as potential fungicidal therapies to overcome or prevent development of fluconazole resistance in Candida species. None of these combinations have been studied clinically except for a heat shock protein 90 inhibitor antibody [43]. Although not addressed in the article, many of the agents being studied have limited clinical application due to drugdrug interactions, QT prolongation, and undesirable side effects including immunosuppression or hypotension.

\section{Molds}

\section{Mucormycosis}

A serious, but relatively uncommon infection primarily seen in immunocompromised hosts, the term mucormycosis encompasses invasive mold infections caused by a wide variety of fungal species with variable sensitivity to available antifungal agents. While therapy is focused largely on surgical excision and correction of underlying immunosuppression or acidosis, there are multiple case series and in vitro studies looking to improve morbidity and mortality with antifungal therapy, either with mono- or CAF therapy. AmB products remain the mainstay of antifungal management despite their toxicity [44]. Given its severity of disease, CAF therapy offers tremendous appeal, but which combination of drugs offers the best option is largely unknown, with most data from in vitro studies.

In vitro studies show that echinocandins at sub-inhibitory concentrations may have an immunomodulatory effect on a variety of mold species including Rhizopus oryzae, a common agent of mucormycosis, potentially by increasing the amount of $\beta$-glucan exposed on the hyphae and thus triggering increased destruction by neutrophils concordant with the amount of exposed $\beta$-glucan. Rhizopus species that have higher mean inhibitory concentrations (MICs) for echinocandins require increased doses of echinocandin compared to other more sensitive molds in order to detect any benefit [45]. The effect of this CAF (AmB-echinocandin) therapy tested in mouse models of diabetic ketoacidosis (DKA) and neutropenia have shown improved survival when compared to either placebo or monotherapy with amphotericin B or an echinocandin alone. The dose necessary for synergy was variable between echinocandins and quantitative methods to measure fungal burden suggest that the beneficial interaction may not be related purely to fungal killing or clearance [46].

Similarly, mouse models comparing combination L-AmB and posaconazole therapy to posaconazole monotherapy did 
not result in a significant difference in mortality when compared to L-AmB alone [47]. Case reports of successful treatment of invasive mucormycosis in solid organ transplant, neutropenic, and hematologic malignancy patients suggest that human results may not mirror mouse model data, though these data are confounded by selection bias, prior treatment, and widely variable therapies with different doses and surgical approaches $[7,48]$. For example, the iron chelator, deferasirox, in combination with a polyene showed a positive interaction in animal models and case reports, but in a randomized, doubleblinded, placebo controlled study, patients with mucormycosis had a higher mortality at 90 days when deferasirox was used in combination [49]. However, since there was some patient imbalance in this lowly powered study, it is still difficult to understand its potential CAF value despite the only randomized trial for treatment of mucormycosis ever performed. As can be seen from this limited data, the role for CAF therapy in the treatment of mucormycosis is largely unknown, and until further studies are performed, the expense and toxicity of the combinations do not readily support its use, and should not replace appropriate surgical debridement and supportive care.

\section{Scedosporium and Fusarium}

Scedosporium species are soil molds that can cause infection in the setting of trauma or immunosuppression and are often intrinsically resistant to polyenes, echinocandins, and most triazoles [50]. Given the rarity of these infections, high incidence of resistance and poor outcomes, in vitro studies were performed comparing different combinations of current antifungal agents including polyenes, echinocandins, triazoles, and terbinafine to look for synergy against the two most common species, Scedosporium apiospermum and Scedosporium prolificans [51]. There was some possible synergy against $S$. apiospermum with itraconazole plus caspofungin, itraconazole plus terbinafine, or azole plus L-AmB for some isolates. However, S. prolificans was more resistant overall and only 15 to $20 \%$ of isolates showed evidence of hyphal damage without sterilization when combinations of ravuconazole and caspofungin or terbinafine and voriconazole were used. Of note, no evidence of antagonism between agents was observed [51]. There are case reports of successful use of voriconazole and terbinafine for S. apiospermum infections, but again limited by reporting bias and precise unknown benefit of voriconazole monotherapy [52].

Disseminated fusariosis is the second most common invasive mold disease in hematologic malignancy patients after IA with a mortality rate exceeding $50 \%$. Due to its resistance to many of the antifungals including $\mathrm{AmB}$, fusariosis treatment is even more complicated [53]. The only data for CAF therapy is a retrospective analysis of patients with invasive fusariosis from the literature. There were 19 cases of invasive fusariosis infections that used CAF therapy with $\mathrm{AmB}$ and either voriconazole or terbinafine. Seven patients had "a clinical response prior to white blood count recovery." Unfortunately, this analysis excluded patients that died before neutrophil recovery, neglecting an important component in the treatment of these infections and the importance of host factors on clinical outcomes. Therefore in reviewing CAF therapy for rare molds, there is no indication from the data provided that two antifungal drugs are more useful or better than monotherapy. Selection and observation biases strongly influence the choices of therapy and confound the role of the host factors in clinical outcomes. As such, there is no precise recommendation for CAF therapy in this group of invasive fungal diseases.

\section{Conclusion}

CAF therapy for the treatment of serious invasive fungal diseases will remain contentious and will be influenced by ongoing case reports and series and weak clinical studies. That said, there is strong evidence for the use of amphotericin B and 5FC in the treatment of cryptococcal infections in HIV-infected patients. Recent data suggest that the combination of voriconazole and anidulafungin may be beneficial in hematologic malignancy patients with GM positive IA infections with a reduction in mortality. Despite the evidence with these infections, the use of CAF therapy in other patients and those with rare mold infections has not been proven and outcomes are primarily influenced by host factors rather than the antifungal treatment chosen.

\section{Compliance with Ethics Guidelines}

Conflict of Interest All the authors state there are no conflicts of interest to declare.

Human and Animal Rights and Informed Consent This article contains no studies with human or animal subjects performed by the author.

\section{References}

Papers of particular interest, published recently, have been highlighted as:

- Of importance

•. Of major importance

1. Johnson MD, Perfect JR. Use of antifungal combination therapy: agents, order, and timing. Curr Fungal Infect Rep. 2010;4(2):87-95.

2. Lewis RE, Kontoyiannis DP. Rationale for combination antifungal therapy. Pharmacotherapy. 2001;21(8 Pt 2):149S-64.

3. Garbati MA, Alasmari FA, Al-Tannir MA, Tleyjeh IM. The role of combination antifungal therapy in the treatment of invasive aspergillosis: a systematic review. Int J Infect Dis: Int J Infect Dis. 2012;16(2):e76-81. 
4. Ullmann AJ, Akova M, Herbrecht R, Viscoli C, Arendrup MC, Arikan-Akdagli S, et al. ESCMID guideline for the diagnosis and management of Candida diseases 2012: adults with haematological malignancies and after haematopoietic stem cell transplantation (HCT). Clin Microbiol Infect. 2012;18 Suppl 7:53-67.

5.• Marr KA, Schlamm HT, Herbrecht R, Rottinghaus ST, Bow EJ, Cornely OA, et al. Combination antifungal therapy for invasive aspergillosis: a randomized trial. Ann Int Med. 2015;162(2):81-9. First major randomized controlled study to compare combination antifungal therapy to monotherapy using voriconazole with anidulafungin versus voriconazole alone. They used a homogeneous population and showed that overall no difference in mortality. However on a subset analysis suggests that patients with a positive galactomannan result may benefit from combination antifungal therapy.

6. Singh N, Husain S. Aspergillosis in solid organ transplantation. Amer J Transplant. 2013;13 Suppl 4:228-41.

7. Pagano L, Cornely OA, Busca A, Caira M, Cesaro S, Gasbarrino C, et al. Combined antifungal approach for the treatment of invasive mucormycosis in patients with hematologic diseases: a report from the SEIFEM and FUNGISCOPE registries. Haematologica. 2013;98(10):e127-30

8. Panackal AA, Parisini E, Proschan M. Salvage combination antifungal therapy for acute invasive aspergillosis may improve outcomes: a systematic review and meta-analysis. Int J Infect Dis. 2014;28C:80-94. A meta-analysis of clinical studies using combination antifungal therapy in a salvage setting. They could only find 16 studies that met their inclusion criteria over 15 years and suggest that combination antifungal therapy improves survival. Many confounding factors and heterogeneous populations makes interpreting the results very difficult.

9. Martin-Pena A, Aguilar-Guisado M, Espigado I, Cisneros JM. Antifungal combination therapy for invasive aspergillosis. Clin Infect Dis. 2014;59(10):1437-45. Great review paper on antifungal combination therapies. Has excellent tables on previous studies and clinical registration studies.

10. Racil Z, Weinbergerova B, Kocmanova I, Muzik J, Kouba M, Drgona L, et al. Invasive aspergillosis in patients with hematological malignancies in the Czech and Slovak republics: Fungal InfectioN Database (FIND) analysis, 2005-2009. Int J Infect Dis. 2013;17(2):e101-9. A database review within a country. Important that these databases are kept to give a real life evaluation of treatment strategies in clinical settings.

11. Clemons KV, Espiritu M, Parmar R, Stevens DA. Comparative efficacies of conventional amphotericin $b$, liposomal amphotericin B (AmBisome), caspofungin, micafungin, and voriconazole alone and in combination against experimental murine central nervous system aspergillosis. Antimicrob Agents Chemother. 2005;49(12): 4867-75.

12. Clemons KV, Stevens DA. Efficacy of micafungin alone or in combination against experimental pulmonary aspergillosis. Med Mycol. 2006;44(1):69-73.

13. Elefanti A, Mouton JW, Verweij PE, Tsakris A, Zerva L, Meletiadis J. Amphotericin B- and voriconazole-echinocandin combinations against Aspergillus spp.: effect of serum on inhibitory and fungicidal interactions. Antimicrob Agents Chemother. 2013;57(10):4656-63.

14. Katragkou A, McCarthy M, Meletiadis J, Petraitis V, Moradi PW, Strauss GE, et al. In vitro combination of isavuconazole with micafungin or amphotericin B deoxycholate against medically important molds. Antimicrob Agents Chemother. 2014;58(11):6934-7.

15. Lewis RE, Kontoyiannis DP. Micafungin in combination with voriconazole in Aspergillus species: a pharmacodynamic approach for detection of combined antifungal activity in vitro. J Antimicrob Chemother. 2005;56(5):887-92.

16. Petraitis V, Petraitiene R, Hope WW, Meletiadis J, Mickiene D, Hughes JE, et al. Combination therapy in treatment of experimental pulmonary aspergillosis: in vitro and in vivo correlations of the concentration- and dose- dependent interactions between anidulafungin and voriconazole by Bliss independence drug interaction analysis. Antimicrob Agents Chemother. 2009;53(6):238291.

17. Mihu CN, Kassis C, Ramos ER, Jiang Y, Hachem RY, Raad II. Does combination of lipid formulation of amphotericin B and echinocandins improve outcome of invasive aspergillosis in hematological malignancy patients? Cancer. 2010;116(22):5290-6.

18. Reference method for broth dilution antifungal susceptibility testing of filamentous fungi; approved standard 2nd edition. CLSI document M38-A2 Wayne, Pa. Clinical and Laboratory Standards Institute. 2008.

19. Forrest G. Role of antifungal susceptibility testing in patient management. Curr Opin Infect Dis. 2006;19(6):538-43.

20. Jeans AR, Howard SJ, Al-Nakeeb Z, Goodwin J, Gregson L, Warn $\mathrm{PA}$, et al. Combination of voriconazole and anidulafungin for treatment of triazole-resistant Aspergillus fumigatus in an in vitro model of invasive pulmonary aspergillosis. Antimicrob Agents Chemother. 2012;56(10):5180-5.

21. Kirkpatrick WR, Vallor AC, McAtee RK, Ryder NS, Fothergill AW, Rinaldi MG, et al. Combination therapy with terbinafine and amphotericin B in a rabbit model of experimental invasive aspergillosis. Antimicrob Agents Chemother. 2005;49(11):4751-3.

22. Zhang M, Su X, Sun WK, Chen F, Xu XY, Shi Y. Efficacy of the combination of voriconazole and caspofungin in experimental pulmonary aspergillosis by different Aspergillus species. Mycopathologia. 2014;177(1-2):11-8.

23. Lafaurie M, Lapalu J, Raffoux E, Breton B, Lacroix C, Socie G, et al. High rate of breakthrough invasive aspergillosis among patients receiving caspofungin for persistent fever and neutropenia. Clin Microbiol Infect. 2010;16(8):1191-6.

24. Safdar A, Rodriguez G, Zuniga J, Akhrass FA, Pande A. High-dose caspofungin as a component of combination antifungal therapy in 91 patients with neoplastic diseases and hematopoietic stem cell transplantation: a critical review of short-term and long-term adverse events. J. Pharmacy Practice. 2013 Dec 23. A retrospective study to demonstrate safety of high dose caspofungin in combination with voriconazole in hematologic malignancy patients. High mortality rates overall and hyperbilirubinemia. Does not appear to help in treatment.

25. Singh N, Limaye AP, Forrest G, Safdar N, Munoz P, Pursell K, et al. Combination of voriconazole and caspofungin as primary therapy for invasive aspergillosis in solid organ transplant recipients: a prospective, multicenter, observational study. Transplantation. 2006;81(3):320-6.

26. Barchiesi F, Mazzocato S, Mazzanti S, Gesuita R, Skrami E, Fiorentini A, et al. Invasive aspergillosis in liver transplant recipients: Epidemiology, clinical characteristics, treatment and outcome in 116 cases. Liver Transplant. 2015;21(2):2014-12. Single center review of aspergillus cases in liver transplants over 25 years. Unfortunately, variability in treatment effects over time effected results as both voriconazole monotherapy and combination therapy improved survival.

27. Perfect JR, Dismukes WE, Dromer F, Goldman DL, Graybill JR, Hamill RJ, et al. Clinical practice guidelines for the management of cryptococcal disease: 2010 update by the infectious diseases society of america. Clin Infect Dis. 2010;50(3):291-322.

28.• Day JN, Chau TT, Wolbers M, Mai PP, Dung NT, Mai NH, et al. Combination antifungal therapy for cryptococcal meningitis. New Eng J Med. 2013;368(14):1291-302. Major study showing significant mortality benefit of 5 flucytosine in combination with amphotericin B over other combinations for treating cryptococcal infections in HIV-infected patients. This paper clearly shows the benefit as recommended standard therapy. 
29. Yao ZW, Lu X, Shen C, Lin DF. Comparison of flucytosine and fluconazole combined with amphotericin B for the treatment of HIV-associated cryptococcal meningitis: a systematic review and meta-analysis. Eur J Clin Microbiol Infect Dis. 2014;33(8):133944.

30. Loyse A, Thangaraj H, Easterbrook P, Ford N, Roy M, Chiller T, et al. Cryptococcal meningitis: improving access to essential antifungal medicines in resource-poor countries. Lancet Infect Dis. 2013;13(7):629-37.

31. Muzoora CK, Kabanda T, Ortu G, Ssentamu J, Hearn P, Mwesigye $\mathrm{J}$, et al. Short course amphotericin B with high dose fluconazole for HIV-associated cryptococcal meningitis. J Infect. 2012;64(1):7681.

32. Jackson AT, Nussbaum JC, Phulusa J, Namarika D, Chikasema M, Kanyemba C, et al. A phase II randomized controlled trial adding oral flucytosine to high-dose fluconazole, with short-course amphotericin B, for cryptococcal meningitis. AIDS. 2012;26(11): 1363-70.

33. Milefchik E, Leal MA, Haubrich R, Bozzette SA, Tilles JG, Leedom JM, et al. Fluconazole alone or combined with flucytosine for the treatment of AIDS-associated cryptococcal meningitis. Med Mycol. 2008;46(4):393-5.

34. Harris JR, Lockhart SR, Debess E, Marsden-Haug N, Goldoft M, Wohrle R, et al. Cryptococcus gattii in the United States: clinical aspects of infection with an emerging pathogen. Clin Infect Dis. 2011;53(12):1188-95.

35. Smith RM, Mba-Jonas A, Tourdjman M, Schimek T, DeBess E, Marsden-Haug N, et al. Treatment and outcomes among patients with Cryptococcus gattii infections in the United States Pacific Northwest. PLoS One. 2014;9(2):e88875.

36. Lockhart SR, Iqbal N, Bolden CB, DeBess EE, Marsden-Haug N, Worhle R, et al. Epidemiologic cutoff values for triazole drugs in Cryptococcus gattii: correlation of molecular type and in vitro susceptibility. Diag Microbiol Infect Dis. 2012;73(2):144-8.

37. Forrest GN, Bhalla P, DeBess EE, Winthrop KL, Lockhart SR, Mohammadi J, et al. Cryptococcus gattii infection in solid organ transplant recipients: description of Oregon outbreak cases. Transplant Infect Dis. 2015 (in press). The emergence of Cryptococcus gattii in this region has demonstrated more virulent activity against organ transplant recipients and is associated with increased fluconazole minimal inhibitory concentrations making step down therapy complicated.

38. Silva EG, Paula CR, Dias AL, Chang MR, Ruiz Lda S, Gambale V, et al. Combination efficacy of voriconazole and amphotericin B in the experimental disease in immunodeficient mice caused by fluconazole-resistant Cryptococcus neoformans. Mycopathologia. 2011;171(4):261-6.

39. Jarvis JN, Meintjes G, Rebe K, Williams GN, Bicanic T, Williams A, et al. Adjunctive interferon-gamma immunotherapy for the treatment of HIV-associated cryptococcal meningitis: a randomized controlled trial. AIDS. 2012;26(9):1105-13.

40. Gamaletsou MN, Sipsas NV, Kontoyiannis DP, Tsiakalos A, Kontos AN, Stefanou I, et al. Successful salvage therapy of refractory HIV-related cryptococcal meningitis with the combination of liposomal amphotericin B, voriconazole, and recombinant interferon-gamma. Diag Microbiol Infect Dis. 2012;74(4):409-11.
41. Rex JH, Pappas PG, Karchmer AW, Sobel J, Edwards JE, Hadley S, et al. A randomized and blinded multicenter trial of high-dose fluconazole plus placebo versus fluconazole plus amphotericin B as therapy for candidemia and its consequences in nonneutropenic subjects. Clin Infect Dis. 2003;36(10):1221-8.

42. Liu S, Hou Y, Chen X, Gao Y, Li H, Sun S. Combination of fluconazole with non-antifungal agents: a promising approach to cope with resistant Candida albicans infections and insight into new antifungal agent discovery. Int J Antimicrob Agents. 2014;43(5): 395-402.

43. Pachl J, Svoboda P, Jacobs F, Vandewoude K, van der Hoven B, Spronk P, et al. A randomized, blinded, multicenter trial of lipidassociated amphotericin B alone versus in combination with an antibody-based inhibitor of heat shock protein 90 in patients with invasive candidiasis. Clin Infect Dis. 2006;42(10):1404-13.

44. Lewis RE, Kontoyiannis DP. Epidemiology and treatment of mucormycosis. Future Microbiol. 2013;8(9):1163-75.

45. Lamaris GA, Lewis RE, Chamilos G, May GS, Safdar A, Walsh TJ, et al. Caspofungin-mediated beta-glucan unmasking and enhancement of human polymorphonuclear neutrophil activity against Aspergillus and non-Aspergillus hyphae. J Infect Dis. 2008;198(2):186-92.

46. Ibrahim AS, Gebremariam T, Fu Y, Edwards Jr JE, Spellberg B. Combination echinocandin-polyene treatment of murine mucormycosis. Antimicrob Agents Chemother. 2008;52(4):1556-8.

47. Ibrahim AS, Gebremariam T, Schwartz JA, Edwards Jr JE, Spellberg B. Posaconazole mono- or combination therapy for treatment of murine zygomycosis. Antimicrob Agents Chemother. 2009;53(2):772-5.

48. Bourke P, Castro P, Rabagliati R, Beltran C, Verdejo H, Winter JL, et al. Zygomycosis over-infection during voriconazole therapy for aspergillosis in a heart transplant patient, successfully treated with liposomal amphotericin and posaconazole. Transpl Infect Dis. 2012;14(5):E56-9.

49. Spellberg B, Ibrahim AS, Chin-Hong PV, Kontoyiannis DP, Morris MI, Perfect JR, et al. The Deferasirox-AmBisome Therapy for Mucormycosis (DEFEAT Mucor) study: a randomized, doubleblinded, placebo-controlled trial. J Antimicrob Chemo. 2012;67(3):715-22.

50. Johnson LS, Shields RK, Clancy CJ. Epidemiology, clinical manifestations, and outcomes of Scedosporium infections among solid organ transplant recipients. Transpl Infect Dis. 2014;16(4):578-87.

51. Cuenca-Estrella M, Alastruey-Izquierdo A, Alcazar-Fuoli L, Bernal-Martinez L, Gomez-Lopez A, Buitrago MJ, et al. In vitro activities of 35 double combinations of antifungal agents against Scedosporium apiospermum and Scedosporium prolificans. Antimicrob Agents Chemother. 2008;52(3):1136-9.

52. Henao-Martinez AF, Castillo-Mancilla JR, Barron MA, Nichol AC. Combination antifungal therapy in the treatment of Scedosporium apiospermum central nervous system infections. Case Rep Infect Dis. 2013;589490.

53. Horn DL, Freifeld AG, Schuster MG, Azie NE, Franks B, Kauffman CA. Treatment and outcomes of invasive fusariosis: review of 65 cases from the PATH Alliance $((\mathrm{R}))$ registry. Mycoses. 2014;57(11):652-8. 